\title{
Interfacial dynamics in transport-limited dissolution
}

\author{
Martin Z. Bazant \\ Department of Mathematics, Massachusetts Institute of Technology, Cambridge, MA 02139
}

(Dated: April 20, 2018)

\begin{abstract}
Various model problems of "transport-limited dissolution" in two dimensions are analyzed using time-dependent conformal maps. For diffusion-limited dissolution (reverse Laplacian growth), several exact solutions are discussed for the smoothing of corrugated surfaces, including the continuous analogs of "internal diffusion-limited aggregation" and "diffusion-limited erosion". A class of non-Laplacian, transport-limited dissolution processes are also considered, which raise the general question of when and where a finite solid will disappear. In a case of dissolution by advectiondiffusion, a tilted ellipse maintains its shape during collapse, as its center of mass drifts obliquely away from the background fluid flow, but other initial shapes have more complicated dynamics.
\end{abstract}

The analysis of interfacial dynamics using conformal maps ("Loewner chains") is a classical subject, which is finding unexpected applications in physics 1, 2, 3]. For dynamics controlled by Laplacian fields, there is a vast literature on continuous models of viscous fingering 4, 5], and stochastic models of diffusion-limited aggregation (DLA) [3, 6] and fractal curves in critical phenomena 1, 2] . Conformal-map dynamics has also been formulated for a class of non-Laplacian growth phenomena of both types 7], driven by conformally invariant transport processes [8]. For growth limited by advection-diffusion in a potential flow, the connection between continuous and stochastic growth patterns has been elucidated [9], and the continuous dynamics has also been studied in cases of freezing in flowing liquids [10, 11, 12, 13].

In all of these examples, the moving interface separates a "solid" region, where singularities in the map reside, a "fluid" region, where the driving transport processes occur and the map is univalent. (In viscous fingering, these are the inviscid and viscous fluid regions, respectively.) Most attention has been paid to problems of "transportlimited growth", where the solid region grows into the fluid region, since the dynamics is unstable and typically leads to cusp singularities in finite time [14, 15, 16] (without surface tension [4]). Here, we consider various time-reversed problems of "transport-limited dissolution" (TLD), where the solid recedes from the fluid region, e.g. driven by advection-diffusion in a potential flow. These are stable processes, so we focus on continuous dynamics, without surface tension.

Stochastic diffusion-limited dissolution (DLD), sometimes called "diffusion-limited erosion" (DLE) or "antiDLA", has been simulated by allowing random walkers in the fluid to annihilate particles of the solid upon contact 17, 19, 20, 21], rather than aggregating as in DLA 18. Outward radial DLE on a lattice, or "internal DLA" (IDLA), where the random walkers start at the origin and cause a fluid cavity to grow in an infinite solid, has also been studied by mathematicians, who proved that the asymptotic shape is a sphere in any dimension 22].

We begin our analysis by summarizing some exact solutions for continuous DLD, which could help to under- stand fluctuations and the long-time limits of DLE and IDLA. Although these solutions are known in somewhat different forms for Laplacian growth [14, 15, 16], it is instructive to summarize them prior to considering problems of TLD by advection-diffusion, to highlight the effects of fluid flow.

Dissolution of surface corrugation. - Let $G(w, t)$ be a conformal map from the left half plane to the fluid region, where steady (Laplacian) diffusion with uniform flux at $-\infty$ drives dissolution of the solid. The interfacial dynamics is given by the (dimensionless) PolubarinovaGalin (PG) equation [3, [5],

$$
\operatorname{Re}\left(\overline{G^{\prime}} G_{t}\right)=1, \quad \text { on } \operatorname{Re} w=0 .
$$

An exact solution has the form,

$$
G(w, t)=w+h(t)+\log \left(1+a(t) e^{w}\right)
$$

with $|a(t)|<1 / 2$ (real) where

$$
\begin{aligned}
a & =\frac{C e^{-t}}{\left(1-a^{2}\right)^{3 / 2}} \sim C e^{-t}+\frac{3}{2} C^{3} e^{-3 t}+\ldots \\
\frac{d h}{d t} & =\frac{1+a-a^{2}}{1-a^{2}}, h \sim t+C\left(e^{-t}-1\right)+\ldots
\end{aligned}
$$

and $C=a(0)\left(1-a(0)^{2}\right)^{3 / 2}$. This solution, shown in Fig. 11 describes the decay of surface corrugations in electropolishing [20, 21, 23, 24] or the displacement of an inviscid fluid by an immiscible viscous fluid in a HeleShaw cell [4, 19? ]. and closely ressembles simulations of "mean-field DLE" (Fig. 2 of [19]).

The linear stability of a flat interface in DLD is contained in the long-time limit of Eqs. (2)-(44). With the dimensions restored ( $w \mapsto w k, G \mapsto G k$, and $t \mapsto k v t)$, the interface shape becomes sinusoidal,

$$
x(y) \sim v t+C e^{-k v t} \cos (k y),
$$

for a Fourier mode of wavenumber $k$ and mean interfacial velocity $v$. We thus recover the classical result 19, 21, 23] that $k v$ is the exponential decay rate of mode $k$, as observed in experiments 24]. 


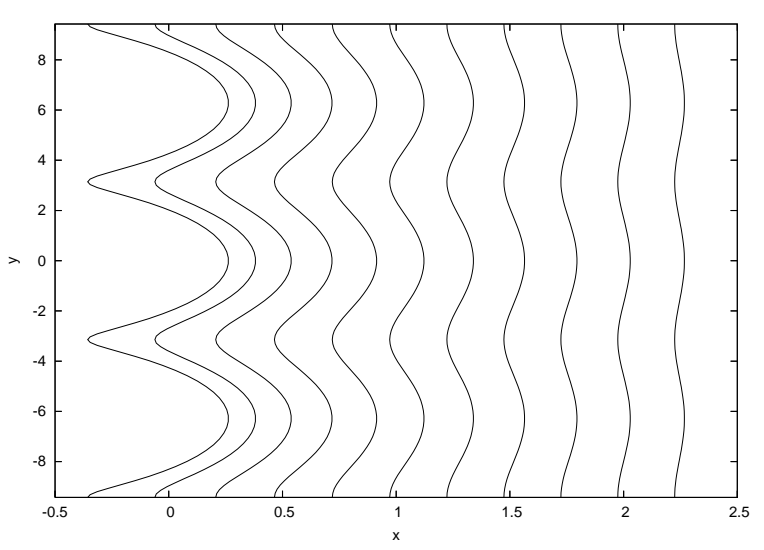

FIG. 1: Diffusion-limited dissolution of a corrugated surface from left to right, for $h(0)=0, a(0)=.3, t=$ $0, .25, .50, \ldots, 2.50$ in Eq. (2).

Outward dissolution of clover-like shapes. - Next we consider the continuous analog of IDLA: DLD in a radial geometry driven by a constant diffusive flux from the origin. This could model quasi-steady melting of an infinite solid around a point source of heat, or the injection of a viscous fluid into a Hele-Shaw cell, displacing an inviscid fluid. The radial PG equation is [3, 5],

$$
\operatorname{Re}\left(\overline{w g^{\prime}} g_{t}\right)=1, \quad \text { on }|w|=1,
$$

where $z=g(w, t)$ is a univalent mapping of the interior of the unit disk. A tractable case is the clover-like $(N-1)$ fold perturbation of a circle,

$$
g(w, t)=a_{1}(t) w+a_{N}(t) w^{N}
$$

first analyzed by Meyer for the (time-reversed) HeleShaw problem 14]. For $a_{1}(0)=1$ and $0<c=a_{N}(0)<$ $1 / N$ (real), the solution has the implicit form,

$$
a_{1}^{2}+N a_{N}^{2}=1+N c^{2}+2 t \text { and } a_{1}^{N} a_{N}=c,
$$

Since $a_{1}(t)$ increases to $\infty$ from $a_{1}(0)=1$ and $a_{N}(t)$ decreases to 0 from $a_{N}(0)=c<1 / N<1$, the following recursion converges very quickly,

$$
a_{1}=\sqrt{2 t+1+N c^{2}\left(1-a_{1}^{-2 N}\right)},
$$

and yields asymptotic approximation upon recursive substitution. An example shown in Figure 2 illustrates the rapid smoothing of a four-leafed clover shape.

From Eqs. (8)-(9) we see that the $(N-1)$-fold radial perturbation decays as a power-law, $a_{N} \propto t^{-N / 2}$, in contrast to the exponential decay of perturbations of a flat interface. We conjecture that the stochastic interface in IDLA is asymptotic to the continuous DLD solution above, up to small logarithmic fluctuations 25], where $w^{N}$ is the smallest perturbation in the initial cluster shape. In general, the continuous dynamics of DLD

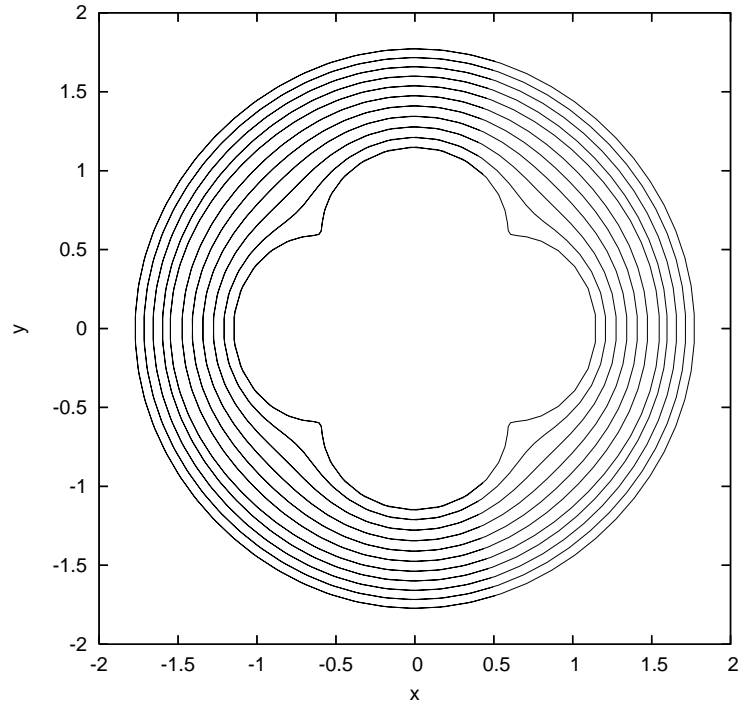

FIG. 2: Outward radial diffusion-limited dissolution driven by a point sink at the origin for an initial four-lobed perturbation of a circle. This exact solution is given by Eqs. (??)-(??) with $N=5, c=0.15, t=0,0.2,0.4, \ldots, 1.0$.

may also accurately approximate the ensemble-averaged stochastic dynamics of IDLA, which is not the case for DLA and other unstable aggregation processes [9].

An (apparently new) explicit solution is possible for $N=2$. In that case, Equation (8) reduces to a depressed cubic [26], $a_{2}^{3}+3 p a_{2}=2 q$, solved by the formula of Cardano and dal Ferro,

$$
a_{1}=\sqrt[3]{q+\sqrt{q^{2}-p^{3}}}+\sqrt[3]{q-\sqrt{q^{2}-p^{3}}} .
$$

where $p=-\left(t+c^{2}+1 / 2\right) / 4$ and $q=-c / 4$.

Inward dissolution of star-like shapes. - Next we briefly consider inward DLD with a constant diffusive flux at infinity, which simply is the time-reverse of Laplacian growth in a radial geometry [5]. Now the map in Eq. (6) must be univalent outside the unit disk, with a Laurent expansion,

$$
g(w, t)=\sum_{n=-\infty}^{1} a_{n}(t) w^{n}, \quad|w| \geq 1
$$

There are well-known solutions for $(N+1)$-fold perturbations [15, 16],

$$
g(w, t)=a_{1}(t) w+a_{-N}(t) w^{-N} \quad \text { for } \quad|w| \geq 1,
$$

where, without loss of generality, $a_{1}(0)=1$ and $a_{-N}(0)=c<1 / N$ is real. Again, the Laurent coeficients satisfy a pair of nonlinear equations, which is most easily solved as a fixed-point iteration,

$$
a_{1}=\sqrt{1-2 t+N c^{2}\left(a_{1}^{2 N}-1\right)} \text { and } a_{-N}=c a_{1}^{N} .
$$




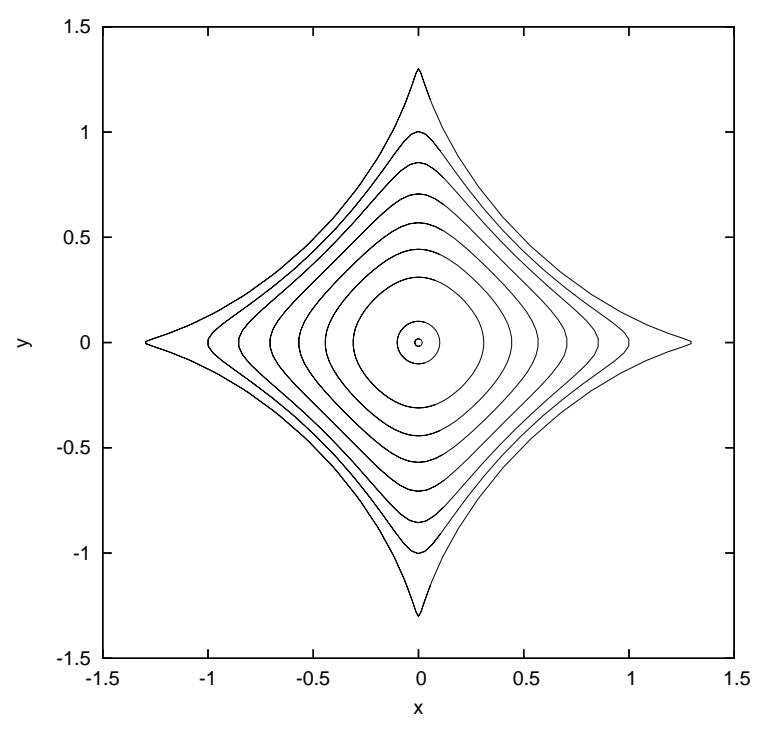

FIG. 3: Inward radial diffusion-limited dissolution driven by a sink at $\infty$ for a four-pointed shape. This exact solution is given by Eqs. (12)-(13) with $N=3, c=0.3$, $t=0,0.04,0.08, \ldots, 0.36,0.3649 ;$ the collapse occurs at $t_{c}=$ 0.365 .

The only qualitative difference with outward DLD is that the solid collapses to a point in a finite time, $t_{c}=(1-$ $\left.N c^{2}\right) / 2$. For $N=1$, an ellipse $(0<c<1)$ or circle $(c=0)$ maintains its shape during collapse,

$$
g(w, t)=\sqrt{1-\frac{t}{t_{c}}}\left(w+\frac{c}{w}\right)
$$

For $N \geq 1$, the shape approaches a circle prior to collapse, according to the asymptotic formula,

$$
a_{1}(t) \sim \sqrt{2\left(t_{c}-t\right)+N c^{2}\left(2\left(t_{c}-t\right)\right)^{N}}
$$

with $a_{-N}(t)=c a_{1}(t)^{N}$. The collapse of a four-pointed shape $(N=3)$ is shown in Figure 3

Advection-diffusion-limited dissolution. - The dynamics of dissolution become more interesting when driven by non-Laplacian (but conformally invariant [8]) transport processes [7], where right-hand side of the PG equation (6) is replaced by the nonuniform, time-dependent flux to the interface, $\sigma(w, t)$. An important example is dissolution by advection-diffusion in a potential flow, e.g. the erosion of rock by flowing water, the evaporation of a fiber coating in a flowing gas, or the melting of a solid column in a flowing liquid. The time-reversed growth problem has been studied extensively in the contexts of freezing [10, 11, 12, 13] and advection-diffusion-limited aggregation [7, 9], but it seems that dissolution - which leads to collapse in finite time - has not been analyzed. For a given initial shape and background flow, when and where will collapse occur?

Consider a finite solid of constant concentration and arbitrary shape in a two dimensional potential flow of zero concentration and uniform velocity far away. The relative importance of advection to diffusion is measured by the Péclet number, $P e_{0}=U L / D$, for a background fluid velocity $U$, diffusivity $D$ and length $L$. The timedependent Péclet number, $P e(t)=P e_{0} a_{1}(t)$, is defined by the conformal radius, $a_{1}(t)$, in Eq. (II). When solid dissolves $\left(a_{1}(t) \rightarrow 0\right)$, diffusion eventually dominates, and it is natural to focus on the low- $P e$ limit.

The flux profile $\sigma(\theta, \mathrm{Pe})$ on the absorber has been studied extensively, and very accurate asymptotic approximations are available 27]. (A numerical code in matlab is also at http://advection-diffusion.net) From the low-Pe approximation,

$$
\sigma \sim \frac{I_{0}(P e)}{K_{0}(P e / 2)} e^{P e \cos \theta}-\mathrm{Pe}\left(\cos \theta+\int_{0}^{P e} d t e^{t \cos \theta} \frac{I_{1}(t)}{t}\right)
$$

which is uniformly accurate in angle $\theta$ up to $P e=10^{-1}$, let us keep only the leading terms,

$$
\sigma \sim \frac{1+P e \cos \theta}{-\log (P e / 4)-\gamma}-P e \cos \theta
$$

where $\gamma=0.577215 \ldots$ is Euler's constant.

In the final stage of collapse where $-\log P e \gg \gamma$, the interface is asymptotically circular, $g(w, t) \sim a_{1}(t) w$. From (17), the radius in this regime satisfies, $a_{1} d a_{1} / d t \sim$ $-1 / \log a_{1}$, and thus has the asymptotic form,

$$
a_{1} \sim \sqrt{\frac{4\left(t-t_{c}\right)}{\log \frac{1}{t-t_{c}}-\log \log \frac{1}{t-t_{c}}+\ldots}}
$$

To describe the dynamics starting at small $P e$ and ending just prior to collapse, it is reasonable to further set $\log P e \approx$ constant. In this intermediate regime, the interfacial dynamics is given by

$$
\operatorname{Re}\left(\overline{w g^{\prime}} g_{t}\right)=-1+b a_{1}(t) \cos \theta \text { for } w=e^{i \theta}
$$

(with a suitable choice of units). The $\cos \theta$ term has the effect of exciting new modes in the shape of the dissolving solid, which are not present in the initial condition. We will see that a circle, $g(w, 0)=w$, translates away from the flow, $g(w, t)=w+a_{0}(t)$, as its upstream side dissolves more quickly prior to collapse. It can also be shown that an $(N+1)$-fold perturbation of a circle (12) will translate and develop an $N$-fold perturbation, $g(w, t)=a_{1}(t) w+a_{0}(t)+a_{-N+1} / w^{N-1}+a_{N}(t) / w^{N}$. Higher Fourier modes in $\sigma(w, t)$ excite additional terms in the Laurent expansion of the shape, $g(w, t)$.

Collapse of a tilted ellipse in a uniform flow. - To illustrate these principles, let us consider a solid ellipse, $g(w, 0)=w+c / w(c<1)$, which is tilted at an angle $\phi=$ $(\arg c) / 2$ with respect to a background flow in the positive $x$ direction. During dissolution, the shape remains an ellipse, but its center of mass, $a_{0}(t)$, move. An example is shown in Fig. [4 


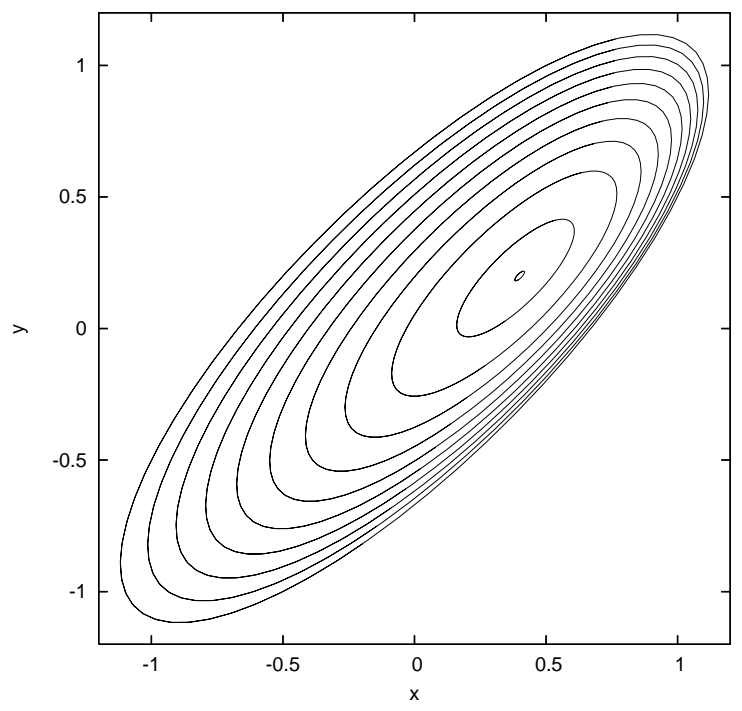

FIG. 4: Inward advection-diffusion-limited dissolution of a tilted elliptical solid in a uniform background flow from left to right $(c=0.5 i, b=0.8, t=0,0.04,0.08, \ldots, 0.36,0.3749)$. The major axis of the ellipse remains oriented at $\pi / 4$, while its center of mass moves at an angle of $\pi / 6$ relative to the background flow. At time $t_{c}=0.375$, the solid disappears at the point $a_{0}\left(t_{c}\right)=0.4+0.2 i$.

The Laurent expansion is given by $g(w, t)=a_{1}(t) w+$ $a_{0}(t)+a_{-1}(t) / w$, where $a_{1}(t)$ is real, but $a_{0}(t)$ and $a_{-1}(t)$, are complex. Substituting into (19) and integrating shows that the conformal has a square-root singularity,

$$
a_{1}(t)=\sqrt{1-\frac{t}{t_{c}}}, \quad t_{c}=\frac{1-|c|^{2}}{2}
$$

since the area decreases linearly to zero (in the approximation of constant total flux): $A(t)=A(0)-2 \pi t$, where $A(0)=\pi\left(1-|c|^{2}\right)$. The collapse time depends on the initial shape through $|c|$. The slowest collapse, $t_{c}=1 / 2$, occurs for a circle, $c=0$, while the collapse time tends to zero for a very elongated ellipse, $|c| \rightarrow 1$, regardless of orientation.

For a constant total flux, the collapse time $t_{c}$ does not depend on the bias introduced by the flow velocity (through $b$ ), although the flow affects the time scale through the initial Péclet number, $P e_{0}$. Mathematically, this is a general consequence of the conformal invariance of the transport process 7], which causes the total flux (Nusselt number) to depend only on the conformal radius and not the asymmetric shape of the particle [27]. Physically, the enhancement of dissolution on the upstream side of the solid is cancelled by the reduction in dissolution on the downstream side.

During dissolution, the ellipse keeps its shape and its orientation with respect to the flow direction since $a_{-1}(t)=c a_{1}(t)$. However, the center of mass moves away from the flow, but also away from the end of the ellipse which protrudes upstream. The velocity of the center of mass is constant and in the $1+c$ direction,

$$
a_{0}(t)=\frac{b(1+c) t}{1-|c|^{2}}
$$

Note that the center of mass does not move along the major axis of the ellipse at angle $\phi$, but instead at an oblique angle $\theta$ given by $\sin \theta=|c| \sin (2 \phi-\theta)$. The final collapse occurs at the point, $a_{0}\left(t_{c}\right)=b(1+c) / 2$. In the simplest case, $c=0$, a circle maintains its shape while its center of mass translates away from the flow at (dimensionless) velocity $b$ until collapse occurs at $x=b / 2$ at time $t_{c}=b / 2$.

For any initial shape, the center of mass will drift away from the flow, as well as away from any protrusions in the direction of the flow. For non-elliptical shapes, it is nontrivial to predict the exact time and place of collapse, even for the simplified dynamics of Eq. (19). For more general transport-limited dynamics 7], predicting the collapse seems like an interesting open problem.

The author thanks J. Propp for an introduction to IDLA.

[1] I. Gruzberg and L. P. Kadanoff, J. Stat. Phys. 114, 1183 (2004).

[2] M. Bauer and D. Bernard, cond-mat/0412372

[3] M. Z. Bazant and D. Crowdy, in Handbook of Materials Modeling, Vol. I, ed. by S. Yip, Art. 4.10 (Springer, 2005).

[4] D. Bensimon, L. P. Kadanoff, S. Liang, B. I. Shraimain, and C. Tang, Rev. Mod. Phys. 58, 977 (1986).

[5] S. D. Howsion, Euro. J. Appl. Math. 3, 209 (1992).

[6] M. Hastings and L. Levitov, Physica D 116, 244 (1998).

[7] M. Z. Bazant, J. Choi, and B. Davidovitch, Phys. Rev. Lett. 91, 04503 (2003).

[8] M. Z. Bazant, Proc. Roy. Soc. Lond. A 460, 1433 (2004).

[9] B. Davidovitch, J. Choi, and M. Z. Bazant, Phys. Rev. Lett. 95, 075504 (2005).

[10] V. A. Maksimov, Prikl. Mat. Mekh. 40, 264 (1976).

[11] K. G. Kornev and V. A. Chugunov, Prikl. Mat. Mekh. 52, 773 (1988).

[12] K. Kornev and G. Mukhamadullina, Proc. Roy. Soc. Lond. A 447, 281 (1994).

[13] M. Alimov, K. Kornev, and G. Mukhamadullina, SIAM J. Appl. Math. 59, 387 (1998).

[14] G. H. Meyer, in Numerical Treatment of Free Boundary Value Problems, p. 202 (Birkhäuser, 1982).

[15] B. I. Shraiman and D. Bensimon, Phys. Rev. A 30, 2840 (1984).

[16] S. D. Howison, SIAM J. Appl. Math. 46, 20 (1986).

[17] L. Paterson, Phys. Rev. Lett. 52, 1621 (1984).

[18] T. Witten and L. M. Sander, Phys. Rev. Lett. 47, 1400 (1981).

[19] C. Tang, Phys. Rev. A 31, 1977 (1985).

[20] P. Meakin and J. M. Deutch, J. Chem Phys. 85, 2320 (1986).

[21] J. Krug and P. Meakin, Phys. Rev. Lett. 66, 703 (1991). 
[22] G. Lawlor, M. Bramson, and D. Griffeath, Ann. Prob. 20, 2117 (1992).

[23] C. Wagner, J. Electrochem. Soc. 101, 225 (1954).

[24] J. Edwards, J. Electrochem. Soc. 100, 189C, 223C (1953).
[25] G. F. Lawlor, Ann. Probab. 23, 71 (1995).

[26] R. W. D. Nickalls, Math. Gazette 77, 354 (1993).

[27] J. Choi, D. Margetis, T. M. Squires, and M. Z. Bazant, J. Fluid Mech. 536, 155 (2005). 